\title{
Pembrolizumab (and friends) in pediatric malignancies: should we consider Hodgkin lymphoma a world of its own?
}

\author{
Mattia Algeri ${ }^{1}$, Franco Locatelli ${ }^{1,2}$ \\ ${ }^{1}$ Department of Pediatric Hematology and Oncology, Scientific Institute for Research and Healthcare (IRCCS), Bambino Gesù Children's Hospital, \\ Rome, Italy; ${ }^{2}$ Sapienza University of Rome, Rome, Italy \\ Correspondence to: Franco Locatelli. Director, Department of Pediatric Hematology and Oncology, IRCCS Ospedale Pediatrico Bambino Gesù, \\ Piazza Sant'Onofrio, 400165 Rome, Italy. Email: franco.locatelli@opbg.net. \\ Provenance and Peer Review: This article was commissioned by the editorial office, Annals of Translational Medicine. The article did not undergo \\ external peer review. \\ Comment on: Geoerger B, Kang HJ, Yalon-Oren M, et al. Pembrolizumab in paediatric patients with advanced melanoma or a PD-L1-positive, \\ advanced, relapsed, or refractory solid tumour or lymphoma (KEYNOTE-051): interim analysis of an open-label, single-arm, phase 1-2 trial. Lancet \\ Oncol 2020;21:121-33.
}

Submitted Mar 26, 2020. Accepted for publication Apr 23, 2020.

doi: $10.21037 / \mathrm{atm} .2020 .04 .54$

View this article at: http://dx.doi.org/10.21037/atm.2020.04.54

Immune checkpoint inhibitors targeting cytotoxic T-lymphocyte associated protein 4 (CTLA-4), PD-L1 and its receptor PD-1 have shown substantial efficacy in several advanced adult malignancies, this resulting into regulatory approval as first- or second-line treatment options (1). Furthermore, several combination approaches have been investigated with notable success in adults, such as simultaneous targeting of PD- 1 and CTLA- 4 in metastatic melanoma and coupling PD-1 inhibition and chemotherapy in non-small-cell lung carcinoma $(2,3)$. By contrast, in the pediatric setting, the clinical development of checkpoint inhibitors is only at the beginning. While survival for childhood leukemia has improved dramatically over the last half-century (4), progress for pediatric solid tumours has been less pronounced and checkpoint inhibitors have been considered as a potential treatment option for relapsed or refractory cases, as well as a strategy to avoid detrimental effects associated with intensive, multimodal chemotherapy. Information regarding safety and efficacy of checkpoint inhibitors in children were scarce until very recently, when first results of three early phase trials, investigating pembrolizumab, atezolizumab and nivolumab respectively, have been published (5-7).

At the end of 2019, Georger and colleagues reported in Lancet Oncology the interim analysis results of KEYNOTE-051, a nonrandomized, open-label, single-arm, multicentre phase I-II trial evaluating pharmacokinetics, pharmacodynamics, toxicity, safety, and anti-tumour activity of the anti-PD-1 antibody pembrolizumab in children (aged between 6 months and 17 years) with various tumour histotypes (5). Eligible patients had histologically or cytologically confirmed advanced melanoma or a PDL1-positive, advanced, relapsed or refractory ( $\mathrm{r} / \mathrm{r})$ solid tumour (including brain neoplasms) or lymphoma with measurable disease according to the Response Evaluation Criteria in Solid Tumours (RECIST) version 1.1. After protocol was amended, enrollment of patients with $\mathrm{r} / \mathrm{r}$ Hodgkin lymphoma (HL) and patients with microsatellite instability-high (MSI-H) tumours was allowed regardless of PD-L1 status. Primary endpoints included: identification of the dose-limiting toxicities at the maximum administered dose, safety and tolerability, and the proportion of patients with objective response to pembrolizumab for each tumour histotype according to the RECIST or the International Neuroblastoma Response Criteria.

In phase I, a modified $3+3$ design (dose-finding) and a modified toxicity probability interval approach were used to determine the pediatric recommended phase II dose. No dose-limiting toxicities were reported in patients treated with the initial dose of pembrolizumab $(2 \mathrm{mg} / \mathrm{kg}$ every 3 weeks) and the observed plasma concentrations at this dose were consistent with those reported in adults 
who received the same dose. Therefore, the pediatric recommended phase II dose of pembrolizumab was established to be $2 \mathrm{mg} / \mathrm{kg}$ every 3 weeks.

After establishment of the recommended phase II dose, anti-tumour activity was evaluated in cohorts of 10 patients for each tumour histotype, expandable to a maximum of 25 patients for those neoplasms in which objective responses were observed. Only children bearing PD-L1 positive tumours (defined as any staining of the stroma or PD-L1 expression on $\geq 1 \%$ of tumour cells by immunohistochemistry) were enrolled, with exception of melanoma, for which PD-L1 expression was not required. Between March 2015 and September 2018, study investigators screened 863 patients. Of them, 796 had tumours evaluable for PD-L1 expression. Among these patients, 278 resulted PD-L1 positive and 155 were enrolled (including 5 patients with PD-L1-negative melanoma and 3 patients with missing PD-L1-status). All 12 patients enrolled in the phase I portion of the study were also included in phase II. Since one patient was not treated with the investigational product because of rapid disease progression, a total of 154 patients received at least one dose of pembrolizumab. Patients were affected by a wide range of tumours, including primary CNS neoplasia, nonCNS solid tumours, sarcomas, Hodgkin and non-HLs. Median age of treated patients was 13 years [interquartile range (IQR), 8-15]. Most patients ( $\mathrm{n}=147)$ had previously received systemic chemotherapy or radiotherapy or both.

The safety profile of pembrolizumab in children was similar to those recorded in the adult population. Treatment-related adverse events (AEs) of any grade were observed in $87(56 \%)$ patients, the most common ones being anemia [in 12 patients (8\%)], fatigue [12 (8\%)], decreased lymphocyte count [11 (7\%)] and pyrexia [11 (7\%)]. Grade III-V AEs considered to be drug-related were recorded in 13 (8\%) patients; decreased lymphocyte count and anemia occurred in $3(2 \%)$ and 2 patients (1\%), respectively. Four patients (3\%) discontinued treatment because of treatment-related AEs. A total of 6 patients (4\%) had AEs that resulted in patient's death, and in two cases these events were considered treatment-related (one case of pneumonitis and pleural effusion and one case of pulmonary oedema during a septic episode).

Concerning efficacy data, pembrolizumab showed significant anti-tumour activity almost exclusively in $\mathrm{r} / \mathrm{r}$ HL. In particular, 9 of 15 evaluable patients with $\mathrm{r} / \mathrm{r} \mathrm{HL}$ achieved an objective response, including two patients who obtained a complete response. These responses were sustained over time, median duration being 17.3 months (95\% CI, 6.5-17.5) and no deaths were recorded in the HL group at data cut off. In post-hoc analyses, the 6- and 12-month progression-free survival were $72.7 \%$ (95\% CI, $42.5-88.8$ ) and $51.9 \%$ (95\% CI, 21.2-75.8), respectively. By contrast, in evaluable patients with any other tumour types $(n=136)$, efficacy signals were found only in few rare subgroups. Thirty patients with other tumour histotypes showed a reduction from baseline in the size of their target lesion, although the majority of these response were of small entity ( $<30 \%$ decrease) and of short duration. Consequently, median progression-free survival of patients with any other tumour except HL was 1.9 months (95\% CI, $1.8-1.9)$. Only 8 cases $(5.9 \%$; $95 \%$ CI, $2.6-11.3)$ of partial response were observed in two patients with adrenocortical carcinoma, two patients with mesothelioma and single patients with malignant ganglioglioma, epithelioid sarcoma, lymphoepithelial carcinoma and malignant rhabdoid tumour. Four of them had ongoing responses at data cutoff. Of note, none of the eight patients with cutaneous melanoma showed an objective responses. This finding is in sharp contrast with data from adult population, in which pembrolizumab is approved for patients with unresectable or metastatic melanoma (8). Since four of the eight patients with melanoma were below the age of 3 years, the authors hypothesized that this lack of efficacy could be, at least in part, explained by the different biology of melanoma in young children (9).

The low efficacy of pembrolizumab as single agent in pediatric solid tumours other than HL is consistent with the results of other trials investigating PD-1/ PD-L1-blockade in the same age group $(6,7)$. Indeed, similarly to what recorded in the KEYNOTE-051 study, monotherapy atezolizumab and nivolumab exhibited very limited activity, despite being tested in a wide spectrum of different pediatric cancers $(6,7)$. In this regard, the main mechanism of action of PD-1/PD-L1 inhibitors is the reinvigoration of $\mathrm{T}$-cell responses against neoantigens presented by MHC class molecules on cancer cell surface. As the number of neoantigens expressed is largely proportional to the number of non-synonymous somatic mutations (NSSMs), the absence of response to checkpoint inhibitors is likely reflecting the low the mutational burden of sporadic pediatric solid tumours (10). Although no information regarding hypermutation status was available in the KEYNOTE-051 interim-report, it has been demonstrated that only $5.5 \%$ of sporadic pediatric solid tumours are hypermutated (defined as $>10$ mutations per 
$\mathrm{Mb})$ (11). Furthermore, the enrichment strategy adopted in the KEYNOTE-051 trial, based on the exclusive enrollment of patients with $\mathrm{PD}-\mathrm{L} 1$ positive tumours, did not provide any additional benefit in terms of clinical responses. This is not surprising since, although it has been reported in adult patients that $\mathrm{PD}-\mathrm{L} 1$ overexpressing tumours have a greater chance to experience pronounced responses to PD-1/ PD-L1-directed therapy, the role of PD-L1 expression as biomarker of response to checkpoint inhibitors is still controversial and confounded by several variables, including different detection antibodies, disparate cutoffs, heterogeneous tissue processing and staining (12). Indeed, no response-predictive biomarkers other than tumour mutational burden have been identified so far for checkpoint inhibitors (13). Results of pharmacodynamic analysis and, especially, tissue-based biological studies, may provide useful insights about the mechanism of action of PD-1 blockade and facilitate a better identification of potential responders. In addition, the role of tumour microenvironment should be carefully evaluated, as it represents a wellrecognized factor influencing, at different levels, the efficacy of immunotherapy in solid malignancies (14). In this regard, the relative lack of information about tumour material obtained before enrollment and during the course of treatment represents a relevant limitation of the report and substantially affects the possibility of obtaining meaningful translational insights from the interim analysis of the KEYNOTE-051 trial. An exploratory biomarker research was planned in the original study design and authors specified that results of these additional secondary analysis will be presented elsewhere. Besides those limitations, the pharmacokinetics and dose-finding analysis performed by Georger and colleagues is of paramount importance, because it has clarified the safety profile and defined the recommended phase II dose of pembrolizumab in children, thus representing the basis upon which future studies should be built.

Undoubtedly, the remarkable efficacy of pembrolizumab in $\mathrm{r} / \mathrm{r} \mathrm{HL}$ is the main finding of the KEYNOTE-051 trial, which deserves greatest attention. The $60 \%$ proportion of durable objective responses observed in this group of patients is superimposable to what observed in larger adult cohorts [overall response rate (ORR) 69\%; 95\% CI, 62.3-75.2\%] (15). Activity signal in HL were also reported in phase I-II studies investigating other PD-1/PD-L1 blocking molecules, atezolizumab and nivolumab, in children and adolescents $(6,7)$. The peculiar sensitivity of HL to checkpoint inhibitors has been well documented in adults and has been attributed to high levels of PD-L1 and PD-L2 expression on ReedSternberg cells, due to amplification of 9 p24.1 region, which includes the CD274 and PDCD1LG2 genes encoding PD-L1 and PD-L2, respectively. Moreover, as the 9p24.1 amplicon also contains the Janus kinase 2 (JAK2) locus, copy number-dependent JAK2-STAT signalling further increases PD-1 ligand expression (16). Bringing together the results of atezolizumab, nivolumab and pembrolizumab trials, the ORR to monotherapy with checkpoint inhibitors in pediatric $\mathrm{HL}$ is $41.1 \%$ (14/34). Although the ORR of pembrolizumab is higher than that reported for atezolizumab (2 partial responses in 9 patients) or nivolumab (1 complete response and 2 partial response in 10 patients), a direct comparison of the three trial is not feasible in these early phases and could lead to misleading conclusion.

Given these preliminary clinical data and biological information, should we therefore consider HL a world of its own when evaluating pembrolizumab (and other PD-1/ PD-L1 blocking molecules) in the pediatric population? The study investigators have in part answered to this question by deciding to continue the enrollment of HL to KEYNOTE-051 trial, in accordance with a dedicated protocol amendment. Results of this separate group will be reported together with those from ongoing cohorts of children with melanoma and MSI-H tumours (5). A detailed description of patient and disease characteristics, including treatment previously received, and a longer follow-up will certainly help better define the role of pembrolizumab in pediatric HL. The results of the extended KEYNOTE-051 trial, however, are likely to represent just the beginning a new era. The encouraging results observed so far in children and young adults with HL, and the solid underlying biological rationale, strongly support the development of further studies in which pembrolizumab, or other PD-1/PD-L1 blockers, are evaluated either alone or in combination with other immunomodulators. In this regard, it is true that large proportions of patients with $\mathrm{r} / \mathrm{r}$ HL can be also rescued by intensive combination chemotherapy, but these salvage regimens typically include anthracyclines, platinum-derivatives, alkylating agents, and/or epipodophyllotoxins, which are associated with significant short- and long-term toxicities and can increase the risk of transplant-related complications. Furthermore, even in the high percentage of patients that are cured with frontline treatment protocols, potentially life-limiting second cancers and treatment-associated cardiovascular dysfunction still represent a matter of concern (17). In light of these considerations, the current major objective 
in pediatric $\mathrm{HL}$ is the development of therapeutic strategies which can ensure high rates of overall survival, while sparing the long-term morbidity and mortality correlated with conventional chemo- radiotherapy schema and, in this perspective, immunotherapy approaches may represent an ideal alternative. Brentuximab vedotin, an antibody-drug conjugate with a CD30-targeting antibody linked to the microtubule-disrupting agent monomethyl auristatin $\mathrm{E}$ by an enzyme-cleavable peptide, has been investigated, either alone or in combination with gemcitabine, in pediatric $\mathrm{r} / \mathrm{r}$ HL, leading to ORR of $47 \%$ (95\% CI, 21-73\%) and $74 \%$ (95\% CI, 58-86\%), respectively $(18,19)$. The combination of Brentuximab vendotin and nivolumab have been evaluated in adults with $\mathrm{r} / \mathrm{r} \mathrm{HL}$, on the basis of their high single-agent activity, being well-tolerated and leading to an impressive objective response rate of $82 \%$ (20). The same combination of Brentuximab vedotin with nivolumab is currently under evaluation also in children and young adults (age range, 5-30 years) with r/r HL (CheckMate 744, NCT02927769). Initial results showed a complete metabolic response in 16 (64\%) of 25 patients and a partial metabolic response in four subjects (16\%) (21). The recent development of CAR $\mathrm{T}$ cell lines targeting CD30 raises, if possible, additional interest towards the immunotherapy approaches in $\mathrm{r} / \mathrm{r} \mathrm{HL}$ $(22,23)$. At the moment, there are no trial investigating the combination of pembrolizumab with other immunotherapies in pediatric HL. However, pembrolizumab is being evaluated in association with chemotherapy in children and young adults with newly diagnosed classical HL who are slow early responders to frontline chemotherapy (NCT03407144). Results of these trials have the potential to revolutionize the current therapeutic approach and might allow patients who are good responders to checkpoint inhibitors and other immune-therapies to avoid treatment with radiotherapy and haemopoietic stem cell transplantation, although the durability of disease control with immunotherapy alone is still an unsolved issue.

In other-than-HL solid tumours, monotherapy with pembrolizumab and other checkpoint inhibitors has yielded, with few exceptions, disappointing results. For this reason, evaluation of PD-1/PD-L1 blocking agents in pediatric tumours should be reserved to selected subtypes in which there is preliminary evidence of efficacy, such as the rare hypermutated cancers, or pursued within combination approaches capable of initiating effective anti-tumour responses. Combination strategies aimed at increasing neoantigen burden with radiotherapy or immunogenic chemotherapy have been investigated in adults, showing significant effect in some cases, but are mainly considered in malignancies responsive to checkpoint inhibitors monotherapy and characterized by a high mutational load (24). In the large proportion of pediatric solid tumours which can be classified as "immunologically cold", redirecting effector $\mathrm{T}$-cells against tumour antigens through bispecific engagers and CAR T cells is theoretically the most attractive approach to generate effective anti-tumour activity and represents, perhaps, the combination setting in which PD-1/PD-L1 blockade has the greatest chances to provide additional benefit, although in a study testing $3^{\text {rd }}$ generation GD2-targeting CAR T cells in neuroblastoma children the addition of pembrolizumab did not increase the response rate (25).

\section{Acknowledgments}

Funding: None.

\section{Footnote}

Conflicts of Interest: Both authors have completed the ICMJE uniform disclosure form (available at http://dx.doi. org/10.21037/atm.2020.04.54). The authors have no conflicts of interest to declare.

Ethical Statement: The authors are accountable for all aspects of the work in ensuring that questions related to the accuracy or integrity of any part of the work are appropriately investigated and resolved.

Open Access Statement: This is an Open Access article distributed in accordance with the Creative Commons Attribution-NonCommercial-NoDerivs 4.0 International License (CC BY-NC-ND 4.0), which permits the noncommercial replication and distribution of the article with the strict proviso that no changes or edits are made and the original work is properly cited (including links to both the formal publication through the relevant DOI and the license). See: https://creativecommons.org/licenses/by-nc-nd/4.0/.

\section{References}

1. Ribas A, Wolchok JD. Cancer immunotherapy using checkpoint blockade. Science 2018;359:1350-5.

2. Gandhi L, Rodríguez-Abreu D, Gadgeel S, et al. Pembrolizumab plus Chemotherapy in Metastatic NonSmall-Cell Lung Cancer. N Engl J Med 2018;378:2078-92. 
3. Larkin J, Chiarion-Sileni V, Gonzalez R, et al. Five-Year Survival with Combined Nivolumab and Ipilimumab in Advanced Melanoma. N Engl J Med 2019;381:1535-46.

4. Hunger SP, Mullighan CG. Acute Lymphoblastic Leukemia in Children. N Engl J Med 2015;373:1541-52.

5. Geoerger B, Kang HJ, Yalon-Oren M, et al. Pembrolizumab in paediatric patients with advanced melanoma or a PD-L1-positive, advanced, relapsed, or refractory solid tumour or lymphoma (KEYNOTE-051): interim analysis of an open-label, single-arm, phase 1-2 trial. Lancet Oncol 2020;21:121-33.

6. Geoerger B, Zwaan CM, Marshall LV, M, et al. Atezolizumab for children and young adults with previously treated solid tumours, non-Hodgkin lymphoma, and Hodgkin lymphoma (iMATRIX): a multicentre phase 1-2 study. Lancet Oncol 2020;21:134-44.

7. Davis KL, Fox E, Merchant MS, et al. Nivolumab in children and young adults with relapsed or refractory solid tumours or lymphoma (ADVL1412): a multicentre, open-label, single-arm, phase 1-2 trial. Lancet Oncol 2020;21:541-50.

8. Chuk MK, Chang JT, Theoret MR, et al. FDA Approval summary: accelerated approval of pembrolizumab for second-line treatment of metastatic melanoma. Clin Cancer Res 2017;23:5666-70.

9. Lu C, Zhang J, Nagahawatte P, et al. The genomic landscape of childhood and adolescent melanoma. J Invest Dermatol 2015;135:816-23.

10. Majzner RG, Heitzeneder S, Mackall CL. Harnessing the Immunotherapy Revolution for the Treatment of Childhood Cancers. Cancer Cell 2017;31:476-85.

11. Campbell BB, Light N, Fabrizio D, et al. Comprehensive Analysis of Hypermutation in Human Cancer. Cell 2017;171:1042-56.e10.

12. Patel SP, Kurzrock R. PD-L1 expression as a predictive biomarker in cancer immunotherapy. Mol Cancer Ther 2015;14:847-56.

13. Chabanon RM, Pedrero M, Lefebvre C, et al. Landscape and Sensitivity to Immune Checkpoint Blockers. Clin Cancer Res 2016;22:4309-21.

14. Binnewies M, Roberts EW, Kersten K, et al. Understanding the tumor immune microenvironment (TIME) for effective therapy. Nat Med 2018;24:541-50.

15. Chen R, Zinzani PL, Fanale MA, et al. Phase II study of the efficacy and safety of pembrolizumab for relapsed/ refractory classic Hodgkin Lymphoma. J Clin Oncol 2017;35:2125-32.

16. Roemer MGM, Advani RH, Ligon AH, et al. PD-
L1 and PD-L2 genetic alterations define classical hodgkin lymphoma and predict outcome. J Clin Oncol 2016;34:2690-7.

17. Mauz-Körholz C, Metzger ML, Kelly KM, et al. Pediatric hodgkin lymphoma. J Clin Oncol 2015;33:2975-85.

18. Locatelli F, Mauz-Koerholz C, Neville K, et al. Brentuximab vedotin for paediatric relapsed or refractory Hodgkin's lymphoma and anaplastic large-cell lymphoma: a multicentre, open-label, phase 1/2 study. Lancet Haematol 2018;5:e450-61.

19. Cole PD, McCarten KM, Pei Q, et al. Brentuximab vedotin with gemcitabine for paediatric and young adult patients with relapsed or refractory Hodgkin's lymphoma (AHOD1221): a Children's Oncology Group, multicentre single-arm, phase 1-2 trial. Lancet Oncol 2018;19:1229-38.

20. Herrera AF, Moskowitz AJ, Bartlett NL, et al. Interim results of brentuximab vedotin in combination with nivolumab in patients with relapsed or refractory Hodgkin lymphoma. Blood 2018;131:1183-94.

21. Harker-Murray P, Leblanc T, Mascarin M, et al. ResponseAdapted Therapy with Nivolumab and Brentuximab Vedotin (BV), Followed By BV and Bendamustine for Suboptimal Response, in Children, Adolescents, and Young Adults with Standard-Risk Relapsed/Refractory Classical Hodgkin Lymphoma. Blood 2018;132:927.

22. Guercio M, Orlando D, Di Cecca S, et al. CD28.OX40 co-stimulatory combination is associated with long in vivo persistence and high activity of CAR.CD30 T cells. Haematologica 2020. doi: 10.3324/haematol.2019.231183.

23. Ramos CA, Ballard B, Zhang H, et al. Clinical and immunological responses after CD30-specific chimeric antigen receptor-redirected lymphocytes. J Clin Invest 2017;127:3462-71.

24. Pearson ADJ, Rossig C, Lesa G, et al. ACCELERATE and European Medicines Agency Paediatric Strategy Forum for medicinal product development of checkpoint inhibitors for use in combination therapy in paediatric patients. Eur J Cancer 2020;127:52-66.

25. Heczey A, Louis CU, Savoldo B, et al. CAR T Cells Administered in Combination with Lymphodepletion and PD-1 Inhibition to Patients with Neuroblastoma. Mol Ther 2017;25:2214-24.

Cite this article as: Algeri M, Locatelli F. Pembrolizumab (and friends) in pediatric malignancies: should we consider Hodgkin lymphoma a world of its own? Ann Transl Med 2020;8(17):1112. doi: $10.21037 /$ atm.2020.04.54 